EPJ Web of Conferences 61, 08005 (2013)

DOI: 10.1051/epjconf/20136108005

(C) Owned by the authors, published by EDP Sciences, 2013

\title{
High-energy signatures of binary supermassive black holes
}

\author{
Gabriela S. Vila ${ }^{1, a}$, Daniela Pérez ${ }^{1, b}$, and Gustavo E. Romero ${ }^{1,2, c}$ \\ ${ }^{1}$ Instituto Argentino de Radioastronomía, C.C. 5, (1894) Villa Elisa, Pcia. de Buenos Aires, Argentina \\ ${ }^{2}$ Facultad de Ciencias Astronómicas y Geofísicas, Paseo del Bosque S/N, (1900) La Plata, Pcia. de Buenos Aires, Argentina
}

\begin{abstract}
Some active galactic nuclei are supposed to harbor binary systems of supermassive black holes. They are an attractive target for gravitational wave experiments. The identification of these systems is mostly based on the detection of periodic features in their light curves. Such identifications, however, are far from being conclusive. In this work we study the perturbations in the emission spectrum of the accretion disk of the primary black hole caused by the orbital motion of the secondary. Then we proceed to determine the kind of spectral energy distributions expected at gamma-ray energies from the inverse Compton up-scattering of disk photons by relativistic electrons in a jet. Gamma-ray observations with instruments such as the future Cherenkov Telescope Array may contribute to a definitive identification of supermassive binary black hole systems at high-energies.
\end{abstract}

\section{Introduction}

Binary systems of supermassive black holes are likely the result of the merger of two galaxies, see e.g. [1, 2]. These objects have recently attracted considerable attention mainly because during the late stages of their evolution they should be intense sources of gravitational waves.

Less than 20 supermassive black hole binaries (SMBHBs) have been discovered up to date but the number of known candidates is much larger. Presently, SMBHBs are usually identified on the basis of periodic patterns in their optical light curves. The best studied case is that of the BL Lac Object OJ 287, that displays outbursts with a periodicity of $\sim 12$ years [3]. The outbursts are though to occur every time a secondary black hole in an eccentric orbit crosses the accretion disk that surrounds a much more massive primary.

Active galactic nuclei (AGN) might as well host at their cores SMBHBs where the orbital plane and the accretion disk of the primary are coplanar. In this particular scenario the secondary would orbit inside the disk and perturb its structure. Depending on the masses of the black holes and the orbital separation, the tidal torques induced by the secondary may clear a gap (i.e. a region of very low mass density) in the disk (e.g. [4-6]). The opening of gaps in disks has been widely studied in the past in the context of planetary migration (see e.g. [7] among many others).

Recently, Kocsis et al. (2012a,b) [8, 9] performed numerical simulations of binary systems of supermassive black holes where the secondary is embedded in the accretion disk of the primary. They calculated the coupled evolution of the disk and the orbit of the secondary under

\footnotetext{
ae-mail: gvila@iar.unlp.edu.ar

be-mail: danielaperez@iar.unlp.edu.ar

$\mathrm{c}_{\mathrm{e}-\mathrm{mail}}$ : romero@iar.unlp.edu.ar
}

the action of tidal and viscous torques and gravitational radiation. An interesting result of these simulations is that, for some combinations of the values of the ratio of masses and the orbital separation, the system settles to a quasisteady state in which an annular gap develops in the disk about the orbital path of the secondary. The flow of matter across the secondary's orbit, however, is not halted, in such a way that there is an inner disk and accretion onto the primary effectively proceeds. Kocsis et al. (2012a,b) named this configuration the "overflowing" regime. The structure of such disk with a gap differs from that of a standard geometrically thin disk [10, 11]. Matter piles up outside the secondary's orbit, so this region becomes hotter and thicker. Assuming that the disk emits as a black body, the increase in the effective temperature must show in its radiative spectrum $[6,9,12]$.

If matter is indeed falling onto the primary, some fraction may be ejected as a pair of relativistic jets. The typical spectral energy distribution (SED) of AGN jets has two main components: a synchrotron peak and an inverse Compton (IC) peak at higher energies. In the case of blazars the IC component extends into the gamma-ray band. The target photons for IC scattering may be provided by the synchrotron radiation field (synchrotron selfCompton, SSC) or may come from outside the jet (external Compton, EC) - from the accretion disk for example. These photons may also absorb the high-energy radiation in its way to the observer. Altogether, then, it is expected that any noticeable feature in the radiative spectrum of the disk has a correlation in the gamma-ray spectrum of the jets.

In this work we consider a SMBHB in the "overflowing" regime defined by Kocsis et al. (2012a,b) where a relativistic jet is launched from the primary black hole. Based on the results of $[8,9]$ we calculate the modified radiative 


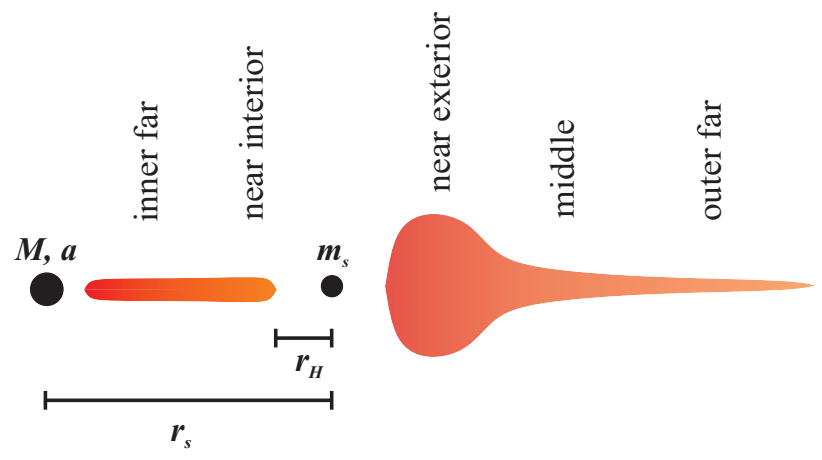

Figure 1. Sketch of a SMBHB where the secondary has cleared an annular gap in the accretion disk. The annotations are explained in the text.

spectrum of the disk. We then investigate the shape of the non-thermal spectrum from the jet to asses if any features are present in the high-energy part of the SED that could reveal the presence of the secondary.

\section{Accretion disk with a gap}

We consider a binary system of supermassive black holes as sketched in Figure 1. The most massive component the primary - is a Kerr black hole of mass $M$ and spin $a=0.99$. The secondary has mass $m_{\mathrm{s}}$ and orbits the primary at a constant distance $r_{\mathrm{s}}$. We define the mass ratio $q \equiv m_{\mathrm{s}} / M \ll 1$. The secondary has cleared an annular gap in the disk. ${ }^{1}$ The half width of the gap is approximately equal to the Hill radius $r_{\mathrm{H}}$ of the secondary, given by

$$
\Delta r_{\text {gap }} \approx r_{\mathrm{H}}=r_{\mathrm{s}}\left(\frac{q}{3}\right)^{1 / 3} \text {. }
$$

According to the results of $[8,9]$ five distinct regions may be identified in an accretion disk in the "overflowing" regime (see Figure 1). In the inner far and outer far regions the disk is basically unperturbed and well-described by the standard model of Shakura \& Sunyaev [10]. To account for the strong gravitational field of the primary, however, we have applied the relativistic version of this model [11] to describe the inner far region. The characteristics of the disk depart from those of a standard disk in the near interior, near exterior and middle regions. In the near interior and near exterior zones the tidal torques exerted on the gas by the secondary dominate over the viscous torques, whereas viscous effects dominate in the middle region. Because of the intense tidal torques a significant amount of gas accumulates outside the secondary's orbit in the near exterior (but also in the middle) zone, heating and inflating the disk. Notice that the simulations of Kocsis et al $(2012 \mathrm{a}, \mathrm{b})$ are not valid inside the gap, so the region $\left[r_{\mathrm{s}}-r_{\mathrm{H}}, r_{\mathrm{s}}+r_{\mathrm{H}}\right]$ is excluded from the calculations.

We have adopted the parametrization provided by Kocsis et al. (2012a,b) for the mass density and surface temperature of the disk as a function of the radius that were

\footnotetext{
${ }^{1}$ We shall not discuss here the conditions for the opening of a steady gap, see [12] for the basic criteria or [7] for a more elaborated discussion.
}

obtained fitting the results of their simulations. We estimated the transition radius between the inner far and near interior regions equating the mass density on both sides, and applied the formula given by Kocsis et al. (2012a) to calculate the transition radius between the near exterior and middle zones. In our models the far zone is usually located so far from the primary that we can safely neglect its contribution to the radiative spectrum of the disk.

We have calculated a number of models in the region of the parameter space where Kocsis et al. (2012a,b) predict that the system settles to the "overflowing" regime. Figure 2 shows the temperature profile as a function of radius $(r=0$ corresponds to the position of $M$ ) and the SED of the disk for a representative model with $M=10^{7} M_{\odot}$, $q=5.5 \times 10^{-3}, r_{\mathrm{s}}=18000 r_{\mathrm{g}}$, and mass accretion rate $\dot{M}=0.1 \dot{M}_{\text {Edd }}$. Here $r_{\mathrm{g}}$ and $\dot{M}_{\text {Edd }}$ are the gravitational radius and the Eddington accretion rate of the primary, respectively. A spiky feature is clearly seen in the temperature profile at the position of the near exterior region. This translates into an increase in the luminosity of the disk at low energies compared to a standard disk.

\section{Jet spectral energy distribution}

The jet model applied here is based on that developed by [13], [14] and [15]. The reader is referred to those works for details on the model; here we only provide a very short account of its basic feature. Two conical relativistic jets are launched perpendicularly to the accretion disk, their symmetry axis forming an angle $\theta_{\text {jet }}$ with the line of sight of the observer. The outflows propagate with constant bulk Lorentz factor $\Gamma_{\text {jet }}$. We assume that a $10 \%$ of the accretion power $\dot{M} c^{2}$ is channeled to the jets. Somewhere along the jets, in the acceleration region, a small fraction $\sim 0.1$ of the jet power is transferred to relativistic particles by some unspecified mechanism - Fermi-like acceleration for instance. Here we consider that only electrons are accelerated. They are injected isotropically in the reference frame co-moving with the jet and follow a power-law distribution in energy. Once injected, electrons cool radiatively and adiabatically as they propagate. Their steady-state distribution in energy and space in the co-moving frame is calculated solving a transport equation that accounts for injection, cooling and convection.

We calculated the broadband non-thermal SED of the jet produced by the interaction of relativistic electrons with the internal magnetic field and the external (disk) and internal (synchrotron) radiation fields. The IC spectra were computed in the Klein-Nisihina regime, and for the external IC we took into account the anisotropy of the disk photon field as seen from the jet (e.g. [16]). Photons that escape the jet may annihilate with another photon from the disk to create an electron-positron pair. We have thus corrected the SEDs by multiplying the luminosity by an attenuation parameter $\exp \left(-\tau_{\gamma \gamma}\right)$, where $\tau_{\gamma \gamma}\left(E_{\gamma}\right)$ is the optical depth for pair production [17]. Notice that this is a first order correction to the SED and that the possible development of an electromagnetic cascade should be further investigated. 

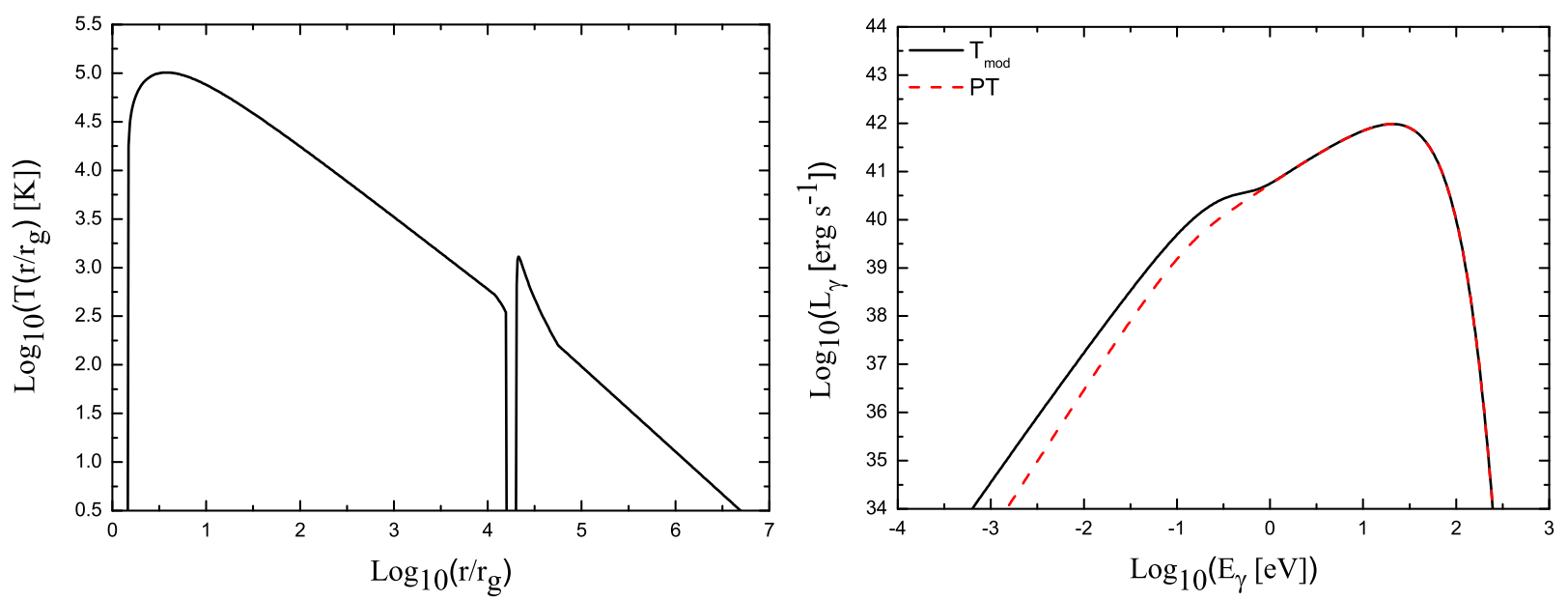

Figure 2. Left. Surface temperature of the disk as a function of radius in units of the gravitational radius of the primary. Right. Solid line: radiative spectrum of the disk (calculated assuming it radiates as a black body at the local surface temperature) for the same set of parameters. Dashed line: spectrum predicted by the relativistic standard disk model of Page \& Thorne (1974) [11].
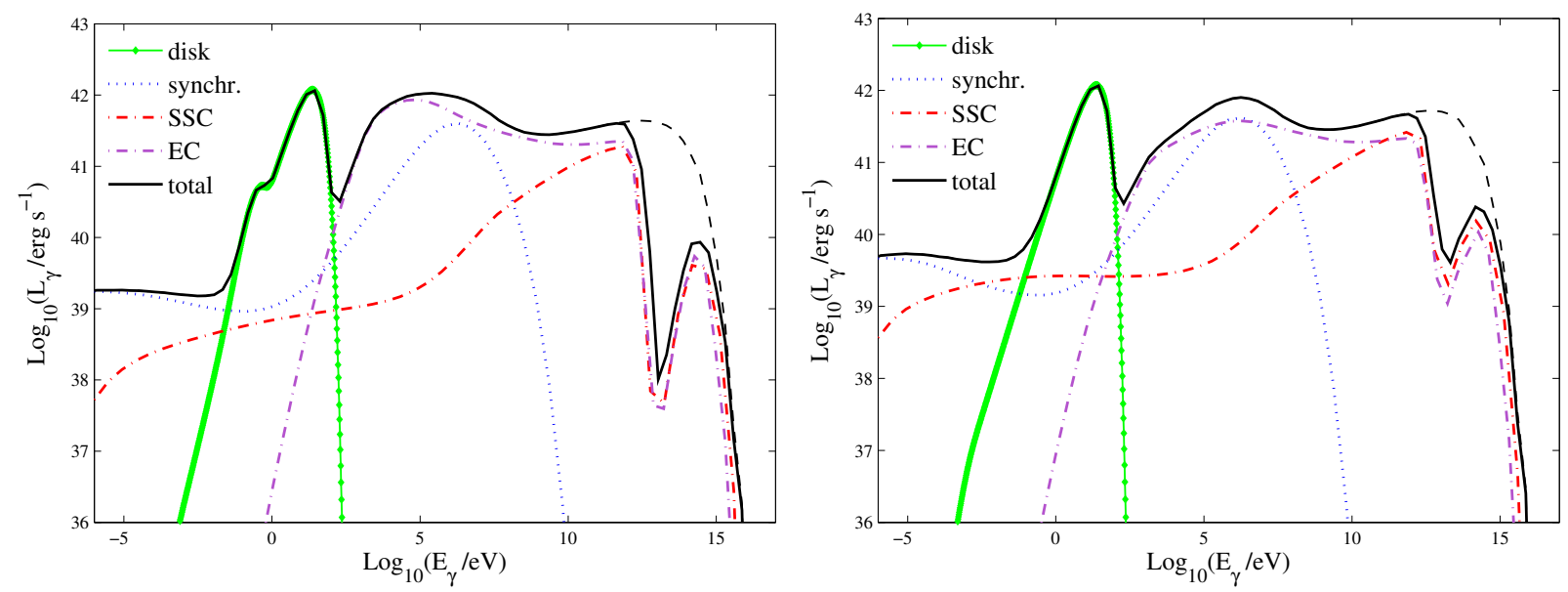

Figure 3. Left. Broadband non-thermal spectral energy distribution of the jet for the case of an accretion disk with a gap. The blackbody emission of the disk is also plotted. The black solid line is the sum of all components multiplied by the attenuation coefficient, whereas the black dashed line is the total luminosity before correcting for absorption. Right. Same but for a disk without a gap.

The radiative spectrum of the jet for the same set of parameters discussed in the previous section is shown in Figure 3. To characterize the jet we adopted $\Gamma_{\text {jet }}=20$, $\theta_{\text {jet }}=5^{\circ}$, and a magnetic field $B \sim 2 \mathrm{G}$ in the acceleration region. For comparison we present the SEDs obtained considering a disk with and without a gap. Basically two elements distinguish them. First, the low energy end of the EC component is slightly more prominent in the model with a disk with a gap. This is the result of the excess of target photons from the near exterior region. The second difference is that, in the same model and for the same reason, the absorption feature at $\sim \mathrm{TeV}$ energies is deeper.

\section{Discussion and perspectives}

The first of the two features described above that differentiate the SEDs, however, appears to be rather weak to be unmistakable recognized. According to the predictions of our model we expect that, besides any feature in the accretion disk spectrum, the characteristics of the emission at very high-energies could lend support to the identification of SMBHB candidates. At those energies, future observations with terrestrial Cherenkov arrays will be the appropriate tool to investigate the kind of effects we predict here.

Some other aspects remain to be analyzed. The calculations must be extended to other values of masses and orbital separations. In particular, we expect the consequences of the existence of the gap to become more pronounced for larger values of $M$. Furthermore, the presence of the secondary may induce precession in the jet of the primary. The EC emission and the absorption effects for a tilted jet could differ from the results presented here. These questions will be addressed in an ongoing expansion of this work. 


\section{Acknowledgments}

GSV thanks the Max-Planck-Institut für Kernphysik in Heidelberg and specially the members of the High Energy Astrophysics Theory Group for their hospitality during the production of this work.

\section{References}

[1] S. Komossa, Mem. S. A. It. 77, 733 (2006)

[2] M. Dotti, A. Sesana, R. Decarli, Adv. Ast. vol. 2012, id. 940568 (2012)

[3] A. Sillanpää, S. Haarala, M. J. Valtonen, B. Sundelius, G. G. Byrd, ApJ 325, 628 (1988)

[4] S. Syer, C. J. Clarke, MNRAS 277, 758 (1995)

[5] J. Goodman, J. C. Tan, ApJ 608, 108 (2004)

[6] Y. T. Liu, S. L. Shapiro, PRD 82, 123011 (2010)
[7] D. N. C. Lin, J. Papalaizou, ApJ 309, 846 (1986)

[8] B. Kocsis, Z. Haiman, A. Loeb, MNRAS 427, 2660 (2012a)

[9] B. Kocsis, Z. Haiman, A. Loeb, MNRAS 427, 2680 (2012b)

[10] N. I. Shakura, R. A. Sunyaev, A\&A 24, 337 (1973)

[11] D. N. Page, K. S. Thorne, ApJ 191, 499 (1974)

[12] K. Gültekin, J. M. Millë̈, ApJ 761, 90 (2012)

[13] G. E. Romero, G. S. Vila, A\&A 485, 623 (2008)

[14] G. S. Vila, G. E. Romero, N. A. Casco, A\&A 538, id.A97 (2012)

[15] M. M. Reynoso, M. C. Medina, G. E. Romero, A\&A 531, id.A30 (2011)

[16] C. D. Dermer, R. Schlickeiser, ApJ 416, 458 (1993)

[17] P. A. Becker, M. Kafatos, ApJ 453, 83 (1995) 